\title{
Enhancement of the SPARC90 code capabilities for pool scrubbing under jet injection regimes
}

\author{
C. Berna ${ }^{1}$, A. Escrivá ${ }^{1}$ J. L. Muñoz-Cobo ${ }^{1} \&$ L. E. Herranz ${ }^{2}$ \\ ${ }^{1}$ Instituto de Ingeniería Energética, Universitat Politècnica de València, \\ Spain \\ ${ }^{2}$ CIEMAT, Spain
}

\begin{abstract}
Submerged gaseous jets may have an outstanding relevance in many industrial processes, but are particularly relevant in severe nuclear accident scenarios, like the one happened at the Fukushima Daiichi NPP several years ago. Even though pool scrubbing has been traditionally associated with fission product retention in suppression pools of Boiling Water Reactors (BWR), in which only low injection velocities exist. There are a number of potential scenarios in which fission product trapping in aqueous ponds may play a key role in the attenuation of source terms, this is the case of SGTR core meltdown sequences.

This paper summarizes the update process of the SPARC90 code, it is based on the state-of-the-art equations for jet hydrodynamics and aerosol removal. The key variables used in the modelling are entrainment inception velocity and droplet size, velocity and concentration. An indirect validation of the hydrodynamic modelling has also been conducted, this validation works have been made through comparisons against data from experiments dealing with the decontamination capability of aqueous ponds under representative severe nuclear accident conditions (mainly ARTIST experiments). Improvements and extension of SPARC-Jet are expected in the near future; however, a final model version will require a more extensive and sound database against which to compare.
\end{abstract}

Keywords: model, submerged jet, pool scrubbing, aerosol retention, severe accident. 


\section{Introduction}

Even though pool scrubbing has been traditionally associated with fission product retention in suppression pools of Boiling Water Reactors (BWR), there are a number of potential scenarios, even in PWR reactors, in which fission product trapping in aqueous ponds may play a key role in the attenuation of source term [1]. This is the case of SGTR core meltdown sequences. The relevance of SGTR severe accidents lies in that a direct pathway from the reactor vessel to the environment might be set through the broken tubes, so that radioactive material bypass reactor containment and other engineering safety features. However, as fission products escaping the damaged fuel reach the steam generator, the secondary side might contain water. Whether water level is over the tubes breach, a fraction of the incoming material might be absorbed.

Over the last decade, several international projects have investigated different aspects of source term under anticipated SGTR core melt condition [2]. In addition, to set up a sound database on aerosol retention, deep insights into aerosol behavior have been gained and, as a result, semi-empiric models have been developed, particularly under dry conditions (i.e., no water in the secondary side) [3]. Modeling of aerosol retention in wet scenarios (i.e., submerged tube breaches) has resulted to be extremely complex. On one side, particle-laden gas is anticipated to reach the steam generator at very high velocities resulting in the formation of a submerged jet when entering the secondary side. On the other, all the tubes in the secondary side will presumably affect gas hydrodynamics. These two effects on gas behavior will strongly influence in-pool particle trapping.

Retention in aqueous ponds was heavily investigated in the $80 \mathrm{~s}$ and computation tools, like the codes SUPRA [4], BUSCA [5] and SPARC [6], were developed. Straight application of these tools for SGTR scenarios, however, is not suitable since their injection hydrodynamics is based on what is known as globule injection (i.e., gas enters the liquid at a low/moderate rate and forms a globule within which the gas circulates) and, besides, no consideration was given to the presence of structures within the pool.

This paper summarizes the SPARC90 extension to the jet injection regime (SPARC-Jet code). Characteristic equations of submerged gas jets have been implemented in the code for variables such as entrainment rate, drop size and velocity, etc. As very few data have been produced in the area of gas jets in ponds, many of the expressions chosen have been taken from the annular gas flow regime. Particle removal equations for dominant mechanisms (i.e., inertial impaction and interception) have been included based on the new hydrodynamic description of the gas. Finally, SPARC-Jet performance has been checked and compared to those from SPARC90 through modeling conditions anticipated in SGTR scenarios. It is worth noting that no validation is pursued here since major elements are still missing in the modeling (i.e., presence of submerged structures); however, given the sound database built-up within the SGTR and ARTIST projects, those scenarios have been chosen to set the code-to-code comparison. 


\section{Jet hydrodynamics}

A submerged gas jet is divided in three zones (fig. 1): an initial expansion (gas and pool pressures match each other); a flow establishment region (central velocity in the potential core remains unchanged); and, a fully developed region (potential core vanishes and central jet velocity decreases).

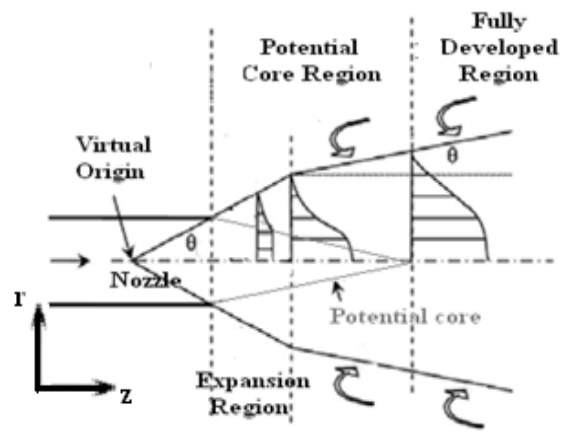

Figure 1: Schematic view of a submerged gas jet.

\subsection{General gas jet characteristics}

The main jet features are here introduced: the jet expansion and developed angle, the end of the expansion zone and the initial jet velocity.

\subsubsection{The expansion angle}

Someya et al. [7] investigated submerged gas jets. They found that a jet large expansion occurred rapidly and extended about $3 \mathrm{~mm}$ from the inlet point; then, expansion progresses at a slower rate (i.e., a narrower angle). The spread angle in the expansion region increased with pressure, whereas the expansion angle in the developed region slightly changed around an average value of about $7^{\circ}$.

\subsubsection{Flow conditions}

The Bubnov model [8] has been chosen to characterize the flow conditions (pressure loss due to sudden expansion considered). According to this model, the critical pressure may be written as:

$$
P_{\text {crit }}=P_{0}\left(\frac{2}{2+C_{\alpha}\left(1-C_{\beta}\right)(\gamma-1)}\right)^{\frac{\gamma}{\gamma-1}}
$$

where $C_{\alpha}$ and $C_{\beta}$ are correction factors of the kinetic energy to account for the pulsating motion of a jet; and $\gamma$ is the isentropic expansion coefficient. The following empirical relation was obtained for a sudden flow expansion:

$$
C_{\alpha}\left(1-C_{\beta}\right)=2.6135-1.4891 \frac{w_{0}}{w_{1}}
$$

where $\mathrm{w}_{0}$ and $\mathrm{w}_{1}$ denote the cross-sectional areas of the narrow and expanded portions of the flow. 
Depending on the flow conditions, different expressions are recommended to estimate gas flow velocity:

1. For critical condition (sonic velocity) $P_{1}<P_{\text {crit }}$

$$
u_{c r i t}=c_{0} \sqrt{\frac{2}{2+C_{\alpha}\left(1-C_{\beta}\right)(\gamma-1)}}
$$

where $c_{0}=\sqrt{\gamma R T_{0}}$.

2. For sub-critical condition (subsonic velocity) $P_{1}>P_{\text {crit }}$

$$
\begin{aligned}
u & =\sqrt{\frac{2 \gamma}{C_{\alpha}\left(1-C_{\beta}\right)(\gamma-1)}\left[\frac{P_{0}}{\rho_{0}}-\frac{P_{1}}{\rho_{1}}\right]}= \\
& =c_{0} \sqrt{\frac{2}{C_{\alpha}\left(1-C_{\beta}\right)(\gamma-1)}\left(1-\left(\frac{p_{1}}{p_{0}}\right)^{\frac{\gamma-1}{\gamma}}\right.}
\end{aligned}
$$

\subsubsection{Jet penetration}

Only a few studies have been made related to a high velocity jets discharging into a liquid pool. Hoefele and Brimacombe [9] gave the following equation to calculate the penetration length for horizontal gas jets for an air/water system:

$$
\frac{L_{j e t}}{D_{0}}=10.7 F^{0.46}\left(\frac{\rho_{g}}{\rho_{l}}\right)^{0.35}
$$

in which Froude number is defined as:

$$
F r_{g}=\frac{\rho_{g} u_{g}^{2}}{\left(\rho_{l}-\rho_{g}\right) g D_{0}}
$$

where $\mathrm{u}_{0}$ and $\mathrm{D}_{0}$ are initial gas jet velocity and nozzle diameter respectively.

\subsection{The entrainment zone}

The entrainment zone is the jet area in which the radial inward flux of the ambient fluid is drawn into the jet. The variables to characterize the gas jet are: entrainment inception velocity, entrainment mass flux and droplets properties.

\subsubsection{Entrainment inception velocity}

The entrainment inception velocity is the gas threshold velocity over which the entrainment process takes place. Several models have been proposed, most of them dependent on fluid properties and on the film Reynolds number:

$$
\operatorname{Re}_{l}=\frac{\rho_{l} u_{l} \delta}{\mu_{l}}
$$

The dominant mechanism of liquid entrainment in low viscous fluids (i.e., water) is shearing-off of roll wave crests. Droplet entrainment would occur when interfacial shear stress exceeds surface tension. Based on the Ishii and Grolmes [10] model, fig. 2 shows that there exists a lower limit of Rel $\left(\mathrm{Re}_{\mathrm{ffoE}}\right)$, under which roll-wave entrainment will not take place (no matter how high the gas 
velocity over the film is). At the other edge, high $\mathrm{Re}_{\mathrm{l}}$, would result in a threshold gas velocity independent of $\mathrm{Re}_{1}$ (rough turbulent regime starts, $\mathrm{Re}_{1} \approx 1500-1750$ ).

A number of models have been published in the open literature. Here the Ishii and Grolmes equations have been selected as they provide a more detailed description of the entrainment domain. It is worth mentioning that all the models found belong to the annular flow regime, so their application to submerged jets is an extrapolation that should be validated.

In horizontal flow and $\operatorname{Re}_{\mathrm{P}}>\operatorname{Re}_{\mathrm{ffOE}}\left(\operatorname{Re}_{\mathrm{ffOE}} \approx 160\right)$ the inception criterion proposed by Ishii is

$$
\begin{gathered}
j_{g} \geq 11.78 N_{\mu}^{0.8} \operatorname{Re}_{l}^{-1 / 3} \frac{\sigma}{\mu_{l}} \sqrt{\frac{\rho_{l}}{\rho_{g}}} \quad \text { for } \quad N_{\mu} \leq \frac{1}{15} ; \operatorname{Re}_{l} \leq 1635 \\
j_{g} \geq 1.35 \operatorname{Re}_{l}^{-1 / 3} \frac{\sigma}{\mu_{l}} \sqrt{\frac{\rho_{l}}{\rho_{g}}} \quad \text { for } \quad N_{\mu}>\frac{1}{15} ; \operatorname{Re}_{l} \leq 1635
\end{gathered}
$$

For the rough turbulent regime $\left(\operatorname{Re}_{1}>1635\right)$ the inception criterion is:

$$
\begin{aligned}
j_{g}>\frac{\sigma}{\mu_{l}} \sqrt{\frac{\rho_{l}}{\rho_{g}}} \times N_{\mu}^{0.8} \quad \text { for } \quad N_{\mu} \leq \frac{1}{15} ; \mathrm{Re}_{l}>1635 \\
j_{g}>\frac{\sigma}{\mu_{l}} \sqrt{\frac{\rho_{l}}{\rho_{g}}} \times 0.1146 \quad \text { for } \quad N_{\mu}>\frac{1}{15} ; \operatorname{Re}_{l}>1635
\end{aligned}
$$

where $\mathrm{j}_{\mathrm{g}}$ is the gas superficial velocity and $\mathrm{N}_{\mu}$ is the viscosity number.

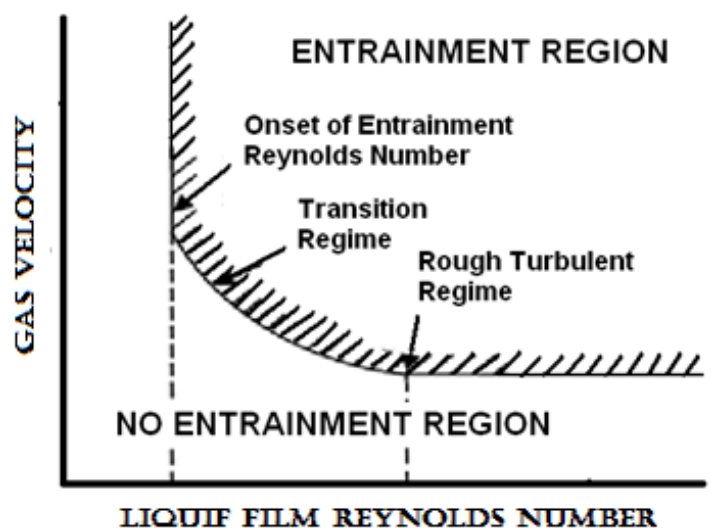

Figure 2: Conceptual sketch of entrainment.

\subsubsection{Gas-liquid interface}

The interfacial shear stress $\left(\tau_{\text {friction }}\right)$ determines the momentum exchange between gas and liquid:

$$
\tau_{\text {friction }}=\frac{1}{2} f_{\text {gi }} \rho_{g}\left(u_{g}-u_{l}\right)^{2}
$$


The gas-liquid interface in a parallel base flow is controlled by the instabilities of Kelvin-Helmholtz generated in inviscid theory by the velocity difference [11]:

$$
u_{l}=u_{g} \sqrt{\frac{\mu_{g}}{\mu_{l}} \frac{\rho_{l}}{\rho_{g}}}
$$

The interfacial friction factor is approximated through Ohnuki's correlation [12].

$$
f_{g i}=1.84 f_{w g} ; f_{w g}=\left\{\begin{array}{l}
\frac{16}{\operatorname{Re}_{g}} ; \operatorname{Re}_{g}<2100 \\
\frac{0.079}{\operatorname{Re}_{g}^{0.25}} ; 2100<\operatorname{Re}_{g}<10^{5} \\
0.0008+\frac{0.05525}{\operatorname{Re}_{g}^{0.237}} ; \operatorname{Re}_{g} \geq 10^{5}
\end{array}\right.
$$

\subsection{Droplet size and velocity profile}

Droplet size can be estimated through the most widely used criteria, which relates the non-dimensional Weber number with a critical empirical value:

$$
W e=\frac{\rho_{g} u_{g}^{2} \phi_{d, \max }}{\sigma}=12
$$

Other similar expressions can be found in the literature [13], but differences result to be minor under anticipated conditions in reactor accident scenarios.

An accurately way to calculate the droplet size is to use an empirical correlation. Due to the fact that no correlations are available for submerged gaseous jets, correlations developed for annular flow can be used, since it is a phenomenology which many similarities. These expressions must be used cautiously, being clear about the conditions in which were developed. We have developed a new expression for horizontal annular flow, which has shown promising results:

$$
\frac{\phi_{v m}}{D}=2.634 \cdot W e_{g}^{-0.23} \mathrm{Re}_{g}^{-0.54} \mathrm{Re}_{l}^{0.13}
$$

The calculation of droplets velocity is done through the Someya et al. measurements [7]:

$$
u_{d}=1.08 P_{0}+3.35
$$

being $\mathrm{P}_{0}$ the stagnation pressure. This equation gives a droplet velocity much smaller than the gas one $(1 / 30-1 / 60)$. Whereas other researchers maintain that droplet velocities are a fraction (0.5-1.0) of gas velocity [13], but all these studies were developed for annular flow.

\subsection{Entrainment mass flux}

Following the way already marked in the previous section, the annular flow correlations are those that best describe the behavior of submerged jets. In the same direction, the expression that we have recently developed is the one which shows better results: 


$$
\frac{E}{1-E}=5.51 \times 10^{-7} \cdot W e_{g}^{2.68} \operatorname{Re}_{g}^{-2.62} \operatorname{Re}_{l}^{0.34}\left(\frac{\rho_{g}}{\rho_{l}}\right)^{-0.37}\left(\frac{\mu_{g}}{\mu_{l}}\right)^{-3.71} C_{W}^{4.24}
$$

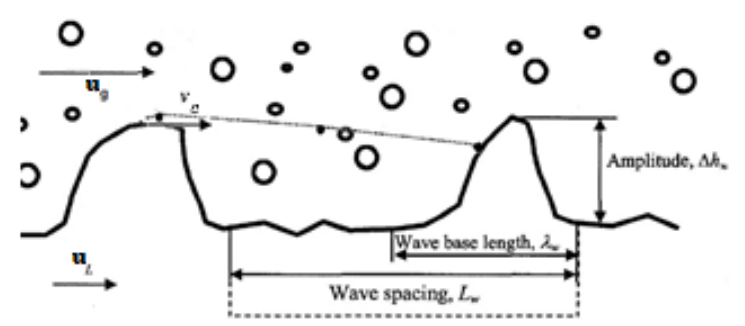

Figure 3: Schematic view of a unit wave.

In the case under study, this expression should be corrected since the above equation was obtained for fully developed flow conditions, which is not the case of a submerged jet (continuous expansion as it moves away from the nozzle). On the one hand, near the nozzle the entrained fraction is far from the equilibrium expression for annular flow, following the path marked by Kataoka et al. [14], a typical form of a relaxation exponential has been chosen (although the coefficient of the exponential has been maximized):

$$
E(z)=\left[1-\exp \left(-10^{-4} \frac{(z / D)^{2} \mathrm{Re}_{l}}{W e_{g}^{0.5}}\right)\right] E
$$

being $\mathrm{z}$ the axial distance to the nozzle and $\mathrm{D}$ the jet diameter. On the other hand, and following the same line, the droplet concentration in the gas decreases exponentially due to deposition processes:

$$
N_{d}(z)=N_{d o} e^{\frac{-\Delta z}{D}}
$$

where $\Delta z$ is the distance from the cell in which the droplet has been dragged from the liquid interface, $\mathrm{N}_{\mathrm{do}}$ is the initial droplet population, $\mathrm{N}_{\mathrm{d}}(\mathrm{z})$ is the droplet concentration at a given distance from the inlet, and $\mathrm{D}$ is the characteristic length of the process that accounts for the droplet motion towards the liquid interface.

\section{Aerosol equations}

Decontamination Factors may be written as a function of the particle collection efficiency $\left(\eta=m_{\text {ret }} / m_{\text {in }}\right)$ :

$$
D F=\frac{1}{1-\eta}
$$

Even though several capturing mechanisms might play a role in the scenario depending on the thermal conditions, at the present stage of the work attention has been focused on those related to droplet-particle mechanical interactions (fig. 4): inertial impaction, interception and Brownian diffusion. Hence, the resulting collection efficiency used is: 


$$
\eta=\eta_{\text {impact }}+\eta_{\text {int ercep }}\left(1-\eta_{\text {impact }}\right)+\eta_{\text {diff }}\left(1-\eta_{\text {impact }}\right)\left\{1-\left[\left(\eta_{\text {int ercep }}\right)\left(1-\eta_{\text {impact }}\right)\right]\right\}
$$

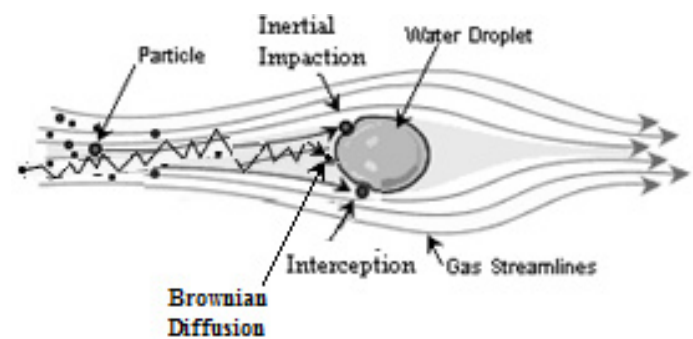

Figure 4: Sketch of droplet-particle mechanical interaction.

\subsection{Inertial impaction}

Heavy particles might be removed from the carrier flow due to sudden changes of direction caused by the presence of obstacles. Particle inertial would make them move away the gas streamlines and eventually collide with the obstacle. Among the different expressions available in the literature, the one proposed by Yung et al. [15] has been chosen:

$$
\eta_{\text {impact }}=\left(\frac{S t k_{p}}{S t k_{p}+0.35}\right)^{2}
$$

where $\mathrm{Stk}_{\mathrm{p}}$ is the particle Stokes number [13],

$$
S t k=\frac{C_{c} \rho_{p} \phi_{p}^{2}\left(u_{g}-u_{d}\right)}{9 \mu \phi_{d}}
$$

and $\mathrm{Cc}$ is the Cunningham slip-correction factor:

$$
C_{c}=1+2.493 \frac{\lambda}{\phi_{p}}+0.84 \frac{\lambda}{\phi_{p}} \exp \left(-0.435 \frac{\phi_{p}}{\lambda}\right)
$$

\subsection{Interception}

Interception takes place when particle radius is larger than distance between the streamline followed by the particle and the obstacle. Jung and Lee [16] proposed:

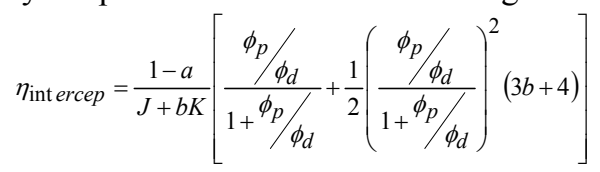

where $a=\left(r_{d} / r_{g}\right)^{3}$ being $\mathrm{r}_{\mathrm{d}}$ is the sphere radius and $\mathrm{r}_{\mathrm{g}}$ is the boundary radius (jet radius); $b=\frac{\mu_{l}}{\mu_{g}} ; J=1-\frac{6}{5} a^{1 / 3}+\frac{1}{5} a^{2}$ and $K=1-\frac{9}{5} a^{1 / 3}+a+\frac{1}{5} a^{2}$.

\subsection{Brownian diffusion}

Brownian motion is the random movement of particles suspended in a fluid. Due to the Brownian diffusion of particles the droplets can capture small particles of 
aerosols by diffusion. The collection efficiency by this diffusion motion is given by [17]:

$$
\eta_{\text {diff }}=\frac{4}{\left(\frac{\mathrm{Re}_{d}}{2}\right) S c_{w}}\left[1+0.4\left(\frac{\mathrm{Re}_{d}}{2}\right)^{1 / 2} S c_{w}^{1 / 3}+0.16\left(\frac{\mathrm{Re}_{d}}{2}\right)^{1 / 2} S c_{w}^{1 / 2}\right]
$$

being the Schmidt number defined as,

$$
S c_{w}=\frac{\mu_{g}}{\rho_{g} D_{\text {diff }}} ; D_{\text {diff }}=\frac{K_{b} T C_{c}}{3 \pi \mu_{g} \phi_{p}}
$$

where $\mathrm{K}_{\mathrm{b}}$ is the Boltzmann constant and $\mathrm{C}_{\mathrm{c}}$ was defined in eqn. (23).

\section{New model implementation}

The SPARC-Jet model has been implemented as a subroutine of the original SPARC90 Fortran code [6]. The SPARC-Jet code is sequentially programmed in two different parts, hydrodynamics and aerosol scrubbing by droplets.

In order to implement the above expression within SPARC90, the jet entrainment zone is split in a number of nodes (fig. 5). In each of them the momentum equation is solved:

$$
\begin{aligned}
& \frac{d}{d z}\left[(1-\alpha(z)) \rho_{l} u_{l}^{2}(z) \pi R^{2}(z)\right]+\frac{d}{d z}\left[\alpha(z) \rho_{g} u_{g}^{2}(z) \pi R^{2}(z)\right] \\
= & {\left[-\frac{d P}{d z} \pi R^{2}(z)-\tau_{\text {friction }} 2 \pi R(z)\right] }
\end{aligned}
$$

Several assumptions have been made: jet conical shape; no phase change and constant velocity along the radial coordinate. The first term in eqn. (26) is the momentum flux of entrained droplets, the second is the gas momentum flux, third term is the pressure loss and fourth term is the friction loss.

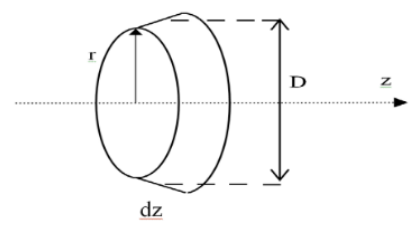

Figure 5: Differential jet node of SPARC-Jet code.

Consistently, DF is estimated as the product of individual contributions of each axial node (i):

$$
D F(k)=\prod_{i=1}^{N} D F_{i}(k) \approx \exp \left(\sum_{i=1}^{N} \gamma(k, z) \cdot \Delta z_{i}\right)
$$

where $\gamma(\mathrm{k}, \mathrm{z})$ can be approximated as:

$$
\gamma(k, z)=\sum_{j=1}^{J}\left[\frac{\pi \phi_{d, j}^{2}(z)}{4} \frac{\left(u_{g}(z)-u_{d, j}(z)\right)}{\alpha(z) u_{g}(z)}\right] \eta_{j, k}(z) N_{d, j}(z)
$$

subscripts $\mathrm{k}$ and $\mathrm{j}$ are the particle and droplet class size. Finally, the total DF is estimated as follows: 


$$
D F=\prod_{k=1}^{K} D F(k)
$$

\section{Experimental SGTR scenarios and DF comparison}

In order to assess the capability of the new SPARC-Jet code, a literature survey on pool scrubbing experiments has been carried out in order to build up a database on jet injection regime. As a result, a total of 8 scenarios have been selected from the ARTIST projects (Table 1). As noted, a wide variety of thermal-hydraulic and aerosol conditions are covered by this set of tests. Even though, because of the non-consideration of condensation processes in the current version of SPARC-Jet, the selected experiments do not contain a significant steam molar fraction.

Table 1: Summary of tests variables and results.

\begin{tabular}{|c|c|c|c|c|c|c|}
\hline Test & A-03 & E-04 & E-07 & E-08 & E-09 & E-10 \\
\hline $1^{\mathrm{ry}}$ pressure (bar) & 1.56 & 1.4 & 1.1 & 1.1 & 4.8 & 4.8 \\
\hline $2^{\text {ry }}$ pressure (bar) & 1.02 & 1.00 & 1.0 & 1.0 & 1.0 & 1.0 \\
\hline $1^{\text {ry }}$ temperature $(\mathrm{K})$ & 303.9 & 297.1 & 330 & 330 & 310 & 313 \\
\hline $2^{\text {ry }}$ temperature $(\mathrm{K})$ & 303.1 & 295.5 & 299 & 299 & 301 & 301 \\
\hline Gas flow rate $(\mathrm{kg} / \mathrm{h})$ & 110.4 & 45.2 & 50 & 50 & 625 & 625 \\
\hline Water submergence $(\mathrm{m})$ & $\begin{array}{l}1.20 \\
2.30 \\
3.60 \\
\end{array}$ & 3.80 & 0.3 & 0.3 & 0.3 & 0.3 \\
\hline Aerosol AMMD $(\mu \mathrm{m})$ & 3.70 & $3.6-4.4$ & 1.4 & 3.7 & 1.4 & 3.7 \\
\hline DF experimental & $\begin{array}{c}124 \\
1251 \\
5739 \\
\end{array}$ & 2097 & 53 & 1370 & 1210 & 2780 \\
\hline DF SPARC90 & $\begin{array}{l}5.236 \\
13.33 \\
28.29\end{array}$ & 57.89 & 1.232 & 1.63 & 1.04 & 1.1 \\
\hline DF SPARC-Jet & $\begin{array}{l}388.7 \\
1332 \\
2366 \\
\end{array}$ & 1401 & 2.179 & 5.283 & 151.5 & 286.2 \\
\hline
\end{tabular}

*For all tests, the break was a guillotine type located $30 \mathrm{~cm}$ above the tube sheet.

Modeling of those conditions with SPARC90 resulted in large discrepancies with experimental measurements. Fig. 6 shows the comparisons of DFs experimental data, SPARC90 and SPARC-Jet version. As noted, SPARC-Jet shows an important improvement of SPARC90 results. Nonetheless, major elements were absent in the modeling, given the lack of any consideration to submerged structures. For this reason, comparisons only have the objective of 
highlight the selection of the right path. Besides, some trends have been captured, for instance, the increase of DF with submergence, aerosol diameter and gas flow rate.

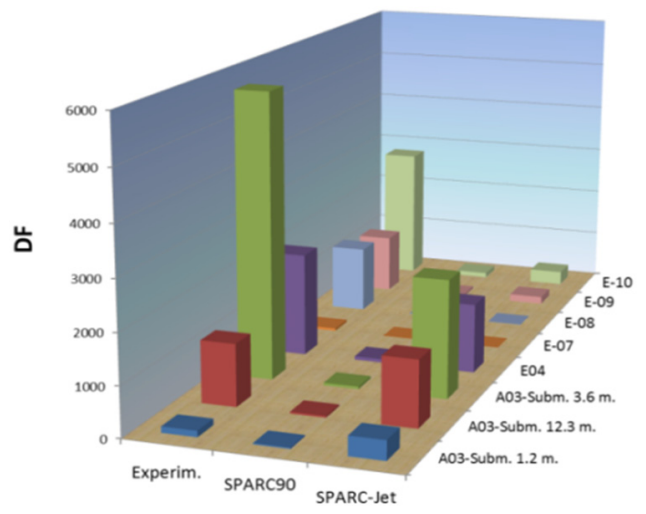

Figure 6: Comparison of the DF results.

\section{Final remarks and further work}

A new version of the SPARC90, focused on submerged high gas velocity injection regime, is under development (SPARC-Jet). An in depth research from the open literature has been carried out, from which jet hydrodynamics has been modelled and aerosol equations has been adopted. Lack of experimental data on "free injection" experiments prevents from setting specific data-prediction comparisons so far, but representative scenarios of jet injection (SGTR core meltdown sequences) have been chosen to serve as a basis for SPARC90 SPARC-Jet comparisons. Even though, the preliminary observations look promising so far, there is still a long path for further development. Some aspects that would be of huge benefit to this work would be:

- Characterization of hydrodynamic equations of submerged jets. Many of the key equations used here were developed in the annular flow regime domain, consequently their validity should be confirmed or other alternative ones should be produced. Particularly relevant are the entrainment/deposition balance of droplets, the droplet-droplet interaction, the droplet characterization and so on.

- Performance of pool scrubbing tests under jet injection, in order to allow validating the SPAR90-Jet predictions.

- The modelling extension to phase change conditions. Processes of steam condensation/evaporation might play an important role by enhancing/ hindering decontamination and by changing droplet dynamics.

- Once jet injection regime will be modelled, its extension to scenarios with submerged structures would still be pending. 


\section{Acknowledgement}

The authors are indebted to the National Plan of I + D by the support of the project MODEXFLAT ENE-2013-48565-C02-01.

\section{References}

[1] Allelein, Auvinen, Ball, Güntay, Herranz, Hidaka, Jones, Kissane, Powers $\&$ Webber "State of the art report on nuclear aerosols". NEA/CSNI/R, 2009.

[2] Güntay S. Suchow D. Debhi A. \& Kapulla R. "ARTIST: Introduction and First Results". Nuclear Engineering and Design, Vol. 231, pp. 109-120, 2004.

[3] Herranz L.E. \& López C. "ARI3SG: Aerosol Retention in the Secondary Side of a Steam Generator. Part I: Model essentials, verification and correlation". Nuclear Engineering and Design, Vol. 248, pp. 270-281, 2012.

[4] Wassel A.T. Mills A.F. \& Bugby D.C. "Analysis of radionuclide retention in water pools". Nuclear Engineering and Design 90(87), 1985.

[5] Ramsdale S. Friederichs H-G. \& Güntay S. "BUSCA JUN91: Reference Manual for the Calculation of Radionuclide Scrubbing in Water Pools". ISBN 3923875665, GRS, 1995.

[6] Owczarski P.C. \& Burk, K.W. "SPARC-90: A Code for Calculating Fission Product Capture in Suppression Pools", 1991.

[7] Someya S. Uchida M. Li Y. Ohshima H. \& Okamoto K. "Entrained droplets in underexpanded gas jet in water". Journal of Visualization, DOI 10.1007/s12650-011-0089-7, 2011.

[8] Bubnov V.A. "Turbulent Isentropic Flows". Journal of Engineering and Thermophysics, Vol. 71, pp. 334-339, 1998.

[9] Hoefele E.O. \& Brimacombe J.K. "Flow Regimes in Submerged Gas Injection”. Metallurgical Transactions, Vol. 10B, pp. 631-648, 1979.

[10] Ishii M. \& Grolmes M.A. "Inception Criteria for Droplet Entrainment in Two-Phase Concurrent Film Flow". AIChE Journal, Vol. 21 pp. 308-318, 1975.

[11] Yecko P. "Viscous modes in two-phase mixing layers". Physics of Fluid, Vol. 14, pp. 4115-4121, 2002.

[12] Spore J.W. et al. "TRAC-M/FORTRAN 90 (Version 3.0) Theory Manual". LA-UR-00-910, July 2000.

[13] Crowe C.T. "Multiphase Flow Handbook (Mechanical Engineering)". CRC Press, Taylor and Francis Group, 2006.

[14] Kataoka I. Ishii M. Nakayama A. "Entrainment and deposition rates of droplets in annular two-phase flow". Int. J. Heat Mass Transf. 43, 15731589, 2000.

[15] Yung S-C. Calvert S. \& Barbarlka H.F. "Venturi Scrubber Performance Model" Environmental Science \& Technology, Vol. 12, No 4, pp. 456459, 1978. 
[16] Jung C.H. \& Lee K.W. "Filtration of fine particles by multiple liquid droplet and gas bubble systems". Aerosol Science and Technology, Vol. 29, pp. 389-401, 1998.

[17] Zhao H. \& Zheng C. "Stochastic Algorithm and Numerical Simulation for Drop Scavenging of Aerosol". Applied Mathematics and Mechanics, Vol. 27, No. 10, pp. 1321-1332, 2006. 